\title{
IDENTIFICATION OF A HELICOPTER DYNAMIC MODEL FOR ACTIVE CONTROL OF VIBRATIONS
}

\author{
S. Bittanti*, P. Bolzern*, P. Colaneri***, P. Delrio**, G. De Nicolao***, \\ F. Lorito $\dagger$, A. Russo** and S. Strada $\dagger$ \\ *Dipartimento di Elettronica, Politecnico di Milano, Piazza Leonardo da Vinci 32. \\ 20133 Milano, Italy \\ **Agusta S.p.a., Cascina Costa-Samarate, Varese, Italy \\ ***Centro di Teoria dei Sistemi, clo Dipartimento di Elettronica, Politecnico di Milano, \\ Piazza Leonardo da Vinci 32, 20133 Milano, Italy \\ †Former Student, Politecnico di Milano, Piazza Leonardo da Vinci 32, 20133 Milano, Italy
}

\begin{abstract}
Motivated by the research on active control of helicopter vibrations, in this paper we present a novel dynamic model of the vibrations induced on the fuselage by the blades. The model provides a small-variation relationship between the swash-plate commands and the vertical force at the rotor hub. Based on data obtained from an Agusta Company simulator, the identified model consists of a time-invariant linear dynamic system complemented with periodically time-varying linear and algebraic blocks.
\end{abstract}

Keywords. Helicopter modeling; Vibration control; System identification; Linear systems; Time-varying systems; Transfer function matrix; Frequency domain.

\section{INTRODUCTION}

The high level of vibrations in helicopters motivates the use of control techniques to reduce such undesired oscillatory phenomena.

The classical mechanical methods to counteract vibration effects, such as appropriate design of the fuselage frame and the location of passive devices (dampers) at critical positions, are becoming insufficient to meet the tight vibration requirements demanded by users. Therefore, a consisten stream of research has recently focused on the development of suitable strategies of active control. Basically, this latter approach consists in superimposing small perturbations of the control variables to the pilot's commands with the purpose of reducing vibrations without affecting the normal flight operations.

Such perturbations are generated in a closed-loop configuration according to two possible approaches, namely the Higher Harmonic Control (HHC) and the Individual Blade Control (IBC). In the IBC method, the pitch angle variations of the blades are controlled independently of each other. On the contrary, the HHC control commands pass through the classical swash-plate actuator so that the pitch angle perturbations are not completely decoupled. In both cases, control design is based on a suitable modellization of the vibration dynamics. The IBC techniques typically require models relating the flapping motion to the pitch angle of a single blade (Ham, 1986; McKillip, 1986). As for the HHC models, they usually consist of small-variation models supplying a linear relationship between input and output harmonics. Due to the intrinsic periodicity of the system, single harmonic inputs generally results into multi-harmonic outputs. Moreover the geometry of the blade system leads to the annihilation of all output harmonics but those at frequencies $\mathrm{kbf}_{0}$, where $\mathrm{k}$ is an integer, $b$ the number of blades and $f_{0}$ the rotation frequency. The relationship between the input harmonics and the output ones is usually referred to as "transfer matrix" in the helicopter jargon (Kretz and Larché, 1979; Davis, 1984; Achache and Gauvrit, 1986; Molusis, 1983; Wood and others, 1983).

In this paper we present a new model of helicopter vibration dynamics suitable for both IBC and HHC strategies. The model partly reflects the physical structure of the system but the single components have been identified by means of a black-box modelling approach. The inputs are the pilot's collective and cyclic commands and the output is the vertical force transmitted by the blades to the rotor hub.

The model is constituted by three parts. The first one is a kinematic description of the swash-plate actuator and allows to compute the pitch angles from the time behavior of the pilot's commands. This part is relevant only in the HHC context. The remaining two parts account for the dynamic relationship between pitch angles and the total hub force in hovering as well as in forward flight condition. To be precise, the second part is a time-invariant dynamic system describing the rotor behavior in hovering condition. The third part, consisting of an algebraic periodic block, is added in order to account for the periodic phenomena arising in forward flight.

In conclusion, the model partly reflects the physical structure of the system. Its output is the vertical force transmitted by the blades to the rotor hub, whereas one can consider as input variables either the pilot's collective and the cyclic commands variations (HHC) or the four pitch angle variations (IBC).

For the identification of the various parts, different techniques have been used, ranging from classical Least Squares fitting for the swash-plate kinematism to parametric identification techniques in frequency domain for the dynamic time-invariant part.

This identification project has been promoted by Agusta S.p.a. under a research contract with the Politecnico di Milano Dipartimento di Elettronica. The specific helicopter dealt with is the Agusta A129. The data used for the identification have been obtained by simulation trials performed on a comples simulation program developed by Agusta S.p.a. A typical 


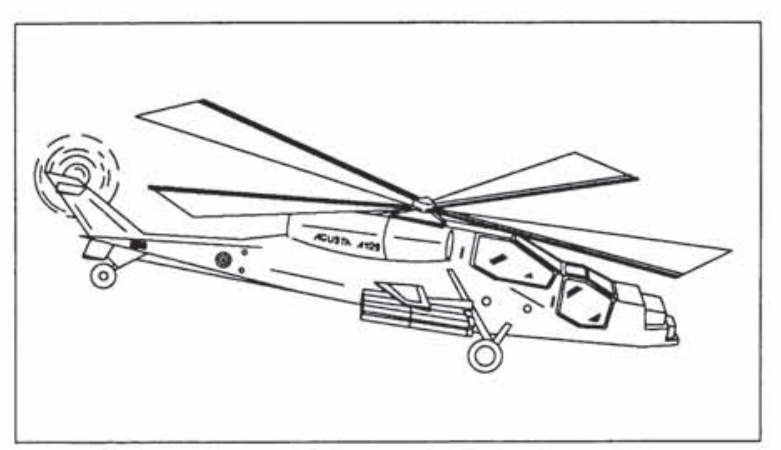

Fig. 1. The Agusta A129 ("Mangusta").

identification trial requires a couple of hours CPU time on a Hitachi NAS-70 XL with vector facility.

The joint research activity, started in 1987, has led to a modellization of the hovering behaviour with a satisfactory fitting of experimental data. Moreover, the model has the advantage of being of relatively small complexity and is suitable for simulation on a Personal Computer, with runs of a few seconds. As for the block concerning the forward flight, its developement is still to be completed.

The paper is organized as follows. First, the system and simulator characteristics are described in Sections 2. The structure of the model to be identified is motivated and discussed in Section 3. Finally, Section 4 presents the results of the identification procedure along with some validation tests.

For easy reference, we now list the symbols used in the paper:

$b=$ number of blades

$f_{0}=$ rotor rotation frequency

$\Omega=$ rotor angular velocity

$T=$ rotor rotation period

$\theta_{i}=i$-th blade pitch angle

$f_{i}=i$ - $t h$ blade share-force (hovering)

$F_{i}=i$-th blade share force (forward flight)

$\mathrm{v}=$ helicopter forward velocity

$\mu=$ helicopter advance ratio

$\varphi_{x}=$ lateral tilt angle of the swash-plate

$\varphi_{y}=$ longitudinal tilt angle of the swash-plate

$h=$ "collective" command of the swash-plate

$R=$ swash-plate radius

$C=$ blade chord

$\Psi_{i}=$ azimuth angle of the $i-t h$ blade.

\section{SYSTEM DESCRIPTION AND SIMULATION}

\subsection{A129 Helicopter}

Agusta A129 "Mangusta" (Fig. 1) is a medium weight (3800 $\mathrm{kg}$ ) helicopter, powered by two turboshaft engines, and accommodates a crew of two. The $11.9 \mathrm{~m}$ diameter main rotor has four fully articulated composite blades rotating at the constant frequency of $5.75 \mathrm{~Hz}$, each with an elastomeric bearing which provides the three articulation functions; blade movements are damped in the rotor plane. The $2.2 \mathrm{~m}$ diameter, two-bladed tail rotor provides the anti-torque force. The maximum operational speed is $270 \mathrm{~km} / \mathrm{h}$.

The helicopter flight is mainly determined by the pitch angles of the blades, which are controlled by the pilot through the so called swash-plate (schematically sketched in Fig. 2). This plate can be shifted along the rotor axis in order to impose the same pitch variation to each blade ("collective pitch") and/or

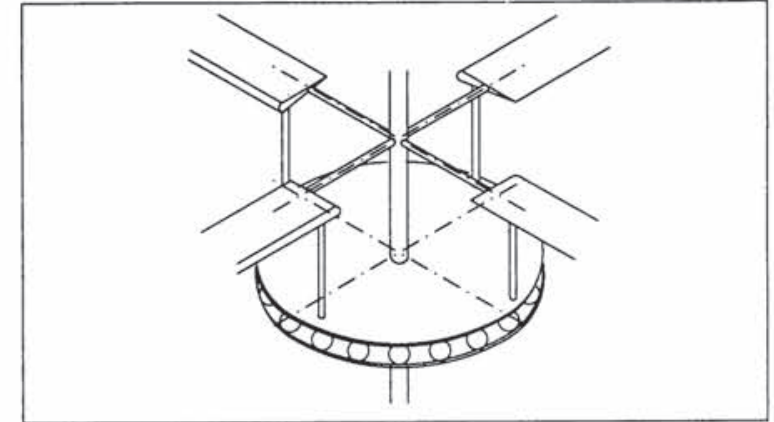

Fig. 2. Swash Plate.

tilted in order to impose periodically varying pitch angles ("cyclic pitch").

When a blade has a non-zero pitch angle, it meets the air with a non-zero angle of attack and allows the generation of aerodynamic forces, which guarantee the necessary thrust either for suspension and vertical flight of the helicopter or for forward flight.

There are two basic flight conditions:

(1) Hovering. The machine is maintained at a fixed position in the air (without forward or lateral movements); this condition is obtained with a "collective" command to the swash-plate. For symmetry, each blade is subject to the same aerodynamic force independently of its angular position. As a consequence, there is a constant vertical thrust at the hub, which balances gravity.

(2) Forward Flight. This flight condition is obtained by tilting the swash-plate in the lateral and/or longitudinal direction. Each blade is then characterized by a periodically varying pitch angle and consequently there is an asymmetry in the rotor behavior depending on the azimuthal position of the blades. Such an asymmetry produces a horizontal component in the total thrust, which determines the forward movement.

\subsection{Helicopter Vibrations}

In Table 1 , the sources of vertical vibrations and their associated frequencies are schematically reported (the rotor fuselage interaction is disregarded). The table refers to four-bladed helicopters. In the sequel, attention will focus on the most important source only, i.e. the main rotor.

The dynamic analysis of the rotor shows that, in steady state and horizontal flight, its aerodynamic forces are transmitted to the fuselage through the hub and the mast according to the

TABLE 1 Vibrations in a Helicopter

\begin{tabular}{|c|c|}
\hline Source & Frequencies, intensity \\
\hline main rotor & $\begin{array}{c}\text { harmonics of frequency } 4 \mathrm{kf}, \\
k \text { integer }\end{array}$ \\
\hline tail rotor & low \\
\hline $\begin{array}{c}\text { geometrical and inertial } \\
\text { asimmetries of the rotor }\end{array}$ & $f_{0}$ \\
\hline aerodynamics & low \\
\hline gusts & low and stochastic \\
\hline $\begin{array}{c}\text { engines, turbines, transmission } \\
\text { compressors, accesories }\end{array}$ & usually high, but known \\
\hline
\end{tabular}




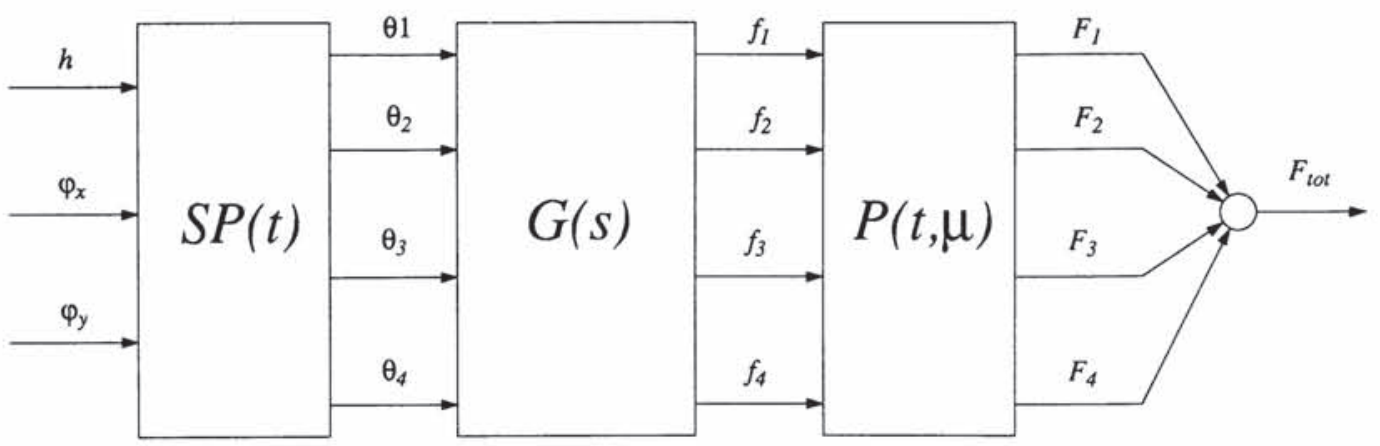

Fig. 3. The model structure.

$\mathbf{S P}(\mathbf{t})$ : periodic algebraic block describing the Swash Plate

G(s) : matrix transfer function (hovering block)

$\mathbf{P}(t, \mu)$ : periodic algebraic block for forward flight effects

following "transformation rules": the harmonics of order $4 k$ of the vertical forces acting on the single blades produce harmonics of the same order of the total vertical force acting on the fuselage; all remaining harmonics cancel at the hub. These transformations are often interpreted as a filtering from the "rotating frame" (the rotor) to the "non-rotating" one (the fuselage). Among all the vertical vibration frequencies, the most noxious is the $4 f_{0}$ one, on which we will concentrate below. It can be shown that the vibration at such a frequency is only influenced by the $3 f_{0}, 4 f_{0}$ and $5 f_{0}$ harmonics in the swash-plate commands. This is why it is important that the model be particularly accurate in the range of frequencies around the interval from $3 f_{0}$ to $5 f_{0}$, i.e. from $18 \mathrm{~Hz}$ to $30 \mathrm{~Hz}$.

\subsection{The Simulator}

The data for the identification have been generated by a complex simulator named NOSTRAN (Russo and Marangione 1988), available at Agusta Company, and running on a Hitachi NAS-70 XL with vector facility. The system simulation program, based on the finite elements method, takes into account aerodynamical, gravitational, elastic, viscous fields so enabling to describe a variety of conditions. One can simulate the effects of gusts, shocks, step and multi-harmonic perturbations. Furthermore, the simulator has been designed so as to easily incorporate active control systems. All variables can be extracted and their time-histories stored for analysis.

In our application, we simulated the A129 rotor in a wind tunnel. Each blade has been modelled as a set of four rigid bodies connected by elastic links.

\section{THE DYNAMIC MODEL FOR VIBRATION CONTROL}

In this Section, the structure of the dynamic model to be identified is introduced. The main objective is to derive a relationship between the swash-plate commands and the vertical force transmitted to the rotor hub by the blades. It is well known that the involved aerodynamic phenomena would require a complex mathematical description in terms of nonlinear, time-varying and partial differential equations. The complexity is such that this model is not apt for the design of simple control systems.
Rather, we aim at deriving an approximate lumped-parameters model able to describe the behavior of the physical system subject to small variations of the input variables in the neighborhood of nominal conditions. Actually, the outputs of the active controller must be small enough not to affect the normal flight operations.

Although the parameter identification will be carried out by means of a black-box approach, the model is structured so as to evidentiate some relevant features of the real system, such as blades interaction and the harmonic filtering effect.

Precisely, the complete model structure is depicted in Fig. 3, where three main blocks can be individuated.

The block $S P(t)$ accounts for the relationship between the pilot's commands and the pitch angles of the four blades due to the presence of the swash-plate. The precise description of this block is deferred to Section 4.

The remaining two blocks describe the influence of the pitch angles $\theta_{i}$ on the four shear-forces $F_{i}(t)$ at the blade-hub. In hovering, the "advance ratio" $\mu=V / \Omega R$ is zero, and the third block reduces to identity so that the dynamics is completely specified by the matrix transfer function $G(s)=\left[G_{i j}(s)\right]$. The off-diagonal elements of $G(s)$ take into account the aerodynamic side-effects caused by a single blade pitch variation on the remaining blades. The assumption of time-invariance is natural in view of the symmetry of the aerodynamic forces pattern in the hovering condition.

On the contrary, when the helicopter is flying forward with constant speed, the asymmetry of the air flow acting on the blades makes the aerodynamic coefficients be periodic. This fact is taken into account in our model by simply cascading $G(s)$ with the algebraic periodic block $P(t, \mu)$ which is a suitable diagonal matrix with periodic entries. $P(t, \mu)$ is such that $P(t, 0)=I$.

In forward flight regime, the four pitch angles $\theta_{i}$ are $\pi / 2$-shifted versions of the same periodic pattern. This is also the case for the shear-forces $F_{i}(t)$. As a consequence, the four periodic gains $P_{i}(t, \mu)$ must satisfy the following constraints:

$$
P_{i+1}(t, \mu)=P_{i}\left(t-\frac{T}{4}, \mu\right)
$$

where $T=1 / f_{0}$ is the rotation period.

Finally, the total force $F$ transmitted to the rotor hub is obtained by summing the four shear-forces $F_{i}$. 


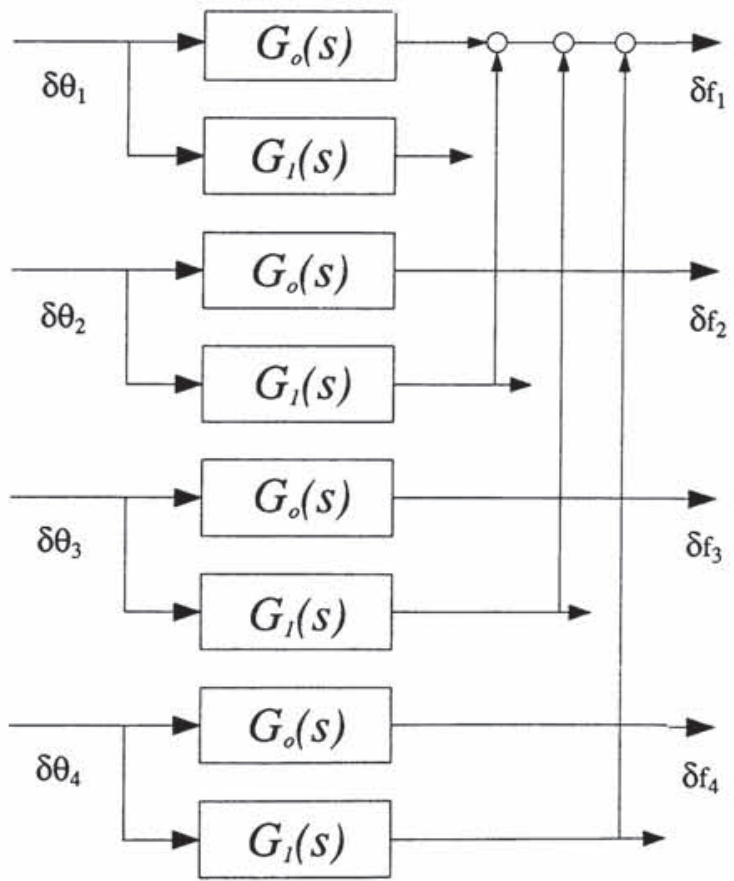

Fig. 4. The Hovering Model.

The proposed model has the advantage of being suitable for the design of vibration controllers based both on the $\mathrm{HHC}$ and the IBC approaches. The latter case can be dealt with by simply removing the swash plate block.

\section{MODEL IDENTIFICATION}

In this section, the different procedures used to identify each block constituting the model will be briefly illustrated.

\subsection{Swash-plate}

This block accounts for the relationship between the swash-plate commands and the pitch angle of each blade $\left(\theta_{1}\right.$ $\left.\theta_{2}, \theta_{3}, \theta_{4}\right)$. Precisely, the commands are the lateral and longitudinal tilt angles $\varphi_{x}$ and $\varphi_{y}$ (producing the so-called "cyclic pitch") and the vertical shift $h$ ("collective pitch").

Such a relationship is expressed by kinematic equations describing the swash-plate and its associated actuators. By considering the simplified mechanical structure of Fig.2, the following equation can be obtained for each blade:

$$
\theta_{i}=-\operatorname{artg}\left[\frac{2 R}{C}\left(\cos \psi_{i} \sin \varphi_{x} \cos \varphi_{y}+\sin \psi_{i} \sin \varphi_{y}\right)+\frac{2}{C} h\right]
$$

where:

$$
\begin{aligned}
& \qquad \Psi_{i}=2 \pi f_{0} t+(i-1) \frac{\pi}{2} \quad \begin{array}{l}
\text { (azimuth angle } \\
\text { of the } i-t h \text { blade) }
\end{array} \\
& C=\text { blade chord } \\
& R=\text { swash plate radius. }
\end{aligned}
$$

Since the equations above do not correspond exactly to the real mechanical layout of the device, it is advisable to identify the parameter of equation (1) by a black box technique rather than relying on their physical meaning. Moreover, as $\varphi_{x}, \varphi_{y}$ and $h$ vary in a very limited range, one can consider a linearized form of equation (1) :

$$
\delta \theta_{i}=A\left(\cos \psi_{i} \delta \varphi_{x}+\sin \psi_{i} \delta \varphi_{y}\right)+B \delta h
$$

where $A=\frac{2 R}{C}, B=\frac{2}{C}$.

Since this equation is linear with respect to A and B a Least Squares technique can be used for parameter identification. The experimental data were obtained from the simulator by choosing suitable values of the variables $\varphi_{x}, \varphi_{y}, h$ and $\psi_{\mathrm{i}}$.

\subsection{Hovering}

This block represents the most important part of the model; under the hypothesis of performing a small-variation analysis, this block can be modelled as a linear dynamic system with four inputs (pitch angles variations: $\delta \theta_{i}, i=1,2,3,4$ ) and four outputs consisting of the variation of the vertical forces transmitted to the hub by the blades $\left(\delta f_{i}, i=1,2,3,4\right)$.

Because of the of the rotor symmetry in hovering, this sub-system can be described by the general matrix equation:

$$
\left[\begin{array}{l}
\delta f_{1}(s) \\
\delta f_{2}(s) \\
\delta f_{3}(s) \\
\delta f_{4}(s)
\end{array}\right]=\left[\begin{array}{llll}
G_{0}(s) & G_{1}(s) & G_{2}(s) & G_{3}(s) \\
G_{3}(s) & G_{0}(s) & G_{1}(s) & G_{2}(s) \\
G_{2}(s) & G_{3}(s) & G_{0}(s) & G_{1}(s) \\
G_{1}(s) & G_{2}(s) & G_{3}(s) & G_{0}(s)
\end{array}\right]\left[\begin{array}{l}
\delta \theta_{1}(s) \\
\delta \theta_{2}(s) \\
\delta \theta_{3}(s) \\
\delta \theta_{4}(s)
\end{array}\right]
$$

$G_{0}(s)$ is the transfer function between the pitch angle imposed to a blade and the vertical force that the same blade transmits to the hub. $G_{1}(s), G_{2}(s), G_{3}(s)$ represent the transfer functions between the pitch angle imposed to a blade and the forces that the other blades transmit to the hub; these function can be interpreted as a mathematical description of the interaction among the blades. A preliminary analysis of the step responses has shown that $G_{1}(s), G_{2}(s), G_{3}(s)$, which describe only secondary effects, can be assumed identical; therefore the block-diagram correspondent to the model becomes the one shown in Fig. 4.

For the identification a frequency-domain technique has been used; In particular, a multi-harmonic pitch variation has been imposed to one blade without perturbing the remaining pitch 
angles; such an input is represented in Fig. 5a and Fig. 5b. From spectral analysis of $\delta f_{1}$ and $\delta f_{2}$ the frequency responses of $G_{0}(s)$ and $G_{1}(s)$ have been estimated. The last step consisted in finding a low order rational approximation able to fit such frequency responses.

The results of the described procedure are given in Figg. 6a, $6 \mathrm{~b}, 6 \mathrm{c}, 6 \mathrm{~d}$; in particular, from the Bode diagrams it is possible to verify the good fitting of the estimated curves to the experimental ones (obtained via the simulator) over the range $0-30 \mathrm{~Hz}$.

\subsection{Forward Flight}

As already said, the identification of the forward flight block is still underway. In this section, we describe the rationale which will be adopted in the estimation procedure.

As mentioned before, the forward flight regime (constant speed), can be obtained superimposing to the "collective" command of each blade, a "cyclic" pitch: it follows that the blades pitch angle is now varying with the azimuth position and the four pitch angles are $\pi / 2$-shifted versions of the same periodic pattern. In forward flight condition the rotor, encountering air with a known velocity, is forward tilted in a stabilized position.

Since the model has to reproduce the filtering effect described in Section 2.2 , it is necessary that, if the same $\pi / 2$-shifted input is given to each blade, the four resulting forces transmitted to the hub must be $\pi / 2$-shifted as well. It is easy to see that the simplest structure for the block $\mathrm{P}(\mathrm{t}, \mu)$ that allows to obtain the mentioned behavior is the following:

$$
P(t, \mu)=\left[\begin{array}{cccc}
P_{1}(t, \mu) & 0 & 0 & 0 \\
0 & P_{2}(t, \mu) & 0 & 0 \\
0 & 0 & P_{3}(t, \mu) & 0 \\
0 & 0 & 0 & P_{4}(t, \mu)
\end{array}\right]
$$

where:

$$
\begin{aligned}
& P_{2}(t, \mu)=P_{1}(t-\tau, \mu) \\
& P_{3}(t, \mu)=P_{1}(t-2 \tau, \mu) \quad \quad, \tau=\mathrm{T} / 4 . \\
& P_{4}(t, \mu)=P_{1}(t-3 \tau, \mu)
\end{aligned}
$$

Therefore, one can focus our attention on the identification of only one among the blocks listed above, say $P_{1}(t, \mu)$

Being $P_{I}(t, \mu)$ a scalar T-periodic function, it can be approximately described by a finite number of its harmonics:

$$
P(t, \mu)=\sum_{-M}^{M} c_{k} e^{j k \Omega t}, \quad \Omega=\frac{2 \pi}{T}
$$

The initial non-parametric problem of estimating a function $\mathrm{P}(\mathrm{t}, \mu)$ has been so reduced to the problem of estimating a finite number of parameters. As input of the algebraic block $\mathrm{P}(\mathrm{t}, \mu)$ a multi-harmonic signal has been taken. The corresponding harmonics of the outputs $\delta \mathrm{F}_{\mathrm{i}}$ can be computed by means of equation (2). The relationship between the input and output harmonics turn out to be a linear one, with parameters which are linear functions of the $c_{i}$ 's. Should the output harmonic be given, one could compute parameters $\left\{c_{i}\right\}$ by resorting to Least-Squares techniques.

The problem is then how to obtain harmonics of $\delta f_{i}$ and $\delta F_{i}$ from experimental data. Such a problem can be tackled as follows. A suitable multi-harmonic pitch angle variation $\delta \theta_{\mathrm{i}}($. has been chosen for one blade, the remaining blades being unperturbed. By imposing such an input both to the Agusta simulator and to the simulator of the (already identified) hovering moriel ( $G(s)$ ), one can obtain both $\delta F_{i}$ and $\delta f_{i}$.

\section{ACKNOWLEDGEMENTS}

This paper has been supported by AGUSTA S.p.A. and Ministero Università e Ricerca Scientifica. The authors gratefully acknowledge Doctors Corrado Chiozzini and Pier Vittorio Ranalletti for discussions and comments.

\section{REFERENCES}

Achache, M.,and M. Gauvrit (1986). Control activ des vibrations sur helicopters par commandes multicycliques autoadaptatifs. Proceedings of the 12th European Rotorcraft Forum.

Davis, M.W. (1984). Development and evaluation of a generic active helicopter vibration controller. Proceedings of the 10th European Rotorcraft Forum.

Ham, N.D. (1986). Helicopter IBC research at MIT 1977-1985. Proceedings of the 12th European Rotorcraft Forum.

Kretz, M.,and M. Larché (1979). Future of Helicopter Rotor Control. Proceedings of the 5th European Rotorcraft Forum.

McKillip, Jr.R.M. (1986). Periodic control of the IBC rotor. Proceedings of the 12th European Rotorcraft Forum.

Molusis, J.A. (1983). The importance of non-linearity on the HHC of helicopter vibrations. 39th Annual Forum of the American Helicopter Society.

Wood, E.R., R.W. Powers, and C.E. Hammond (1983). On developing and flight testing a HHC System. 39th Annual Forum of the American Helicopter Society.

Russo, A., and M. Marangione (1988). NOSTRAN- NOnlinear STRucture ANalysis (User's Manual). Agusta S.p.a. 


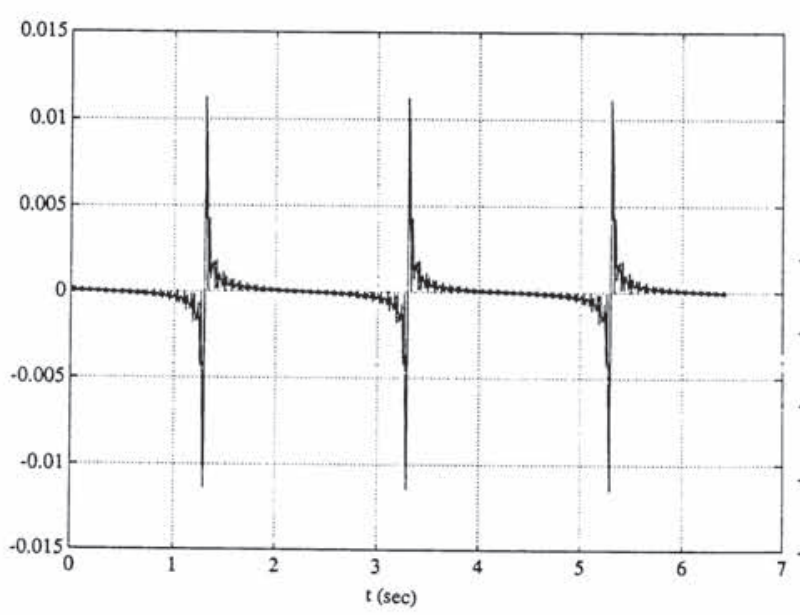

Fig. 5a. Input multi-harmonic signal.

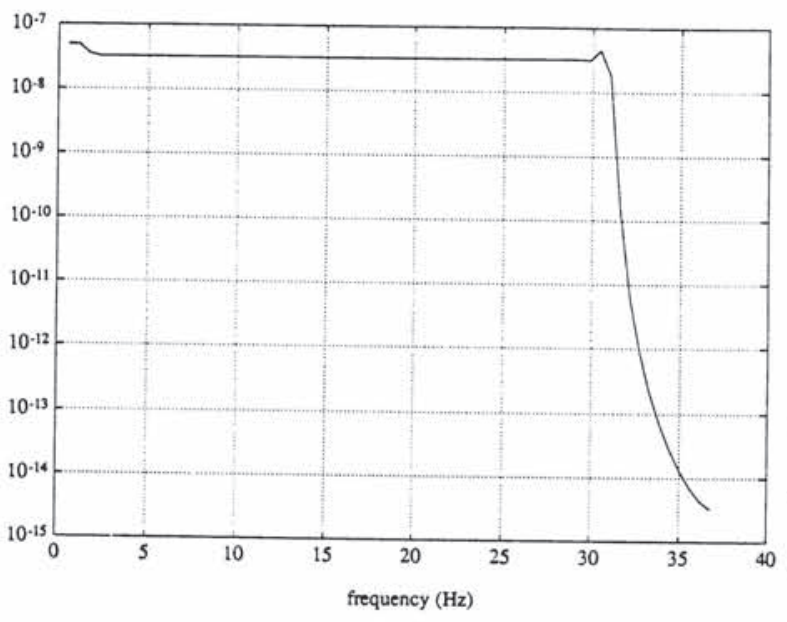

Fig. 5b. Spectrum of the input multi-harmonic signal.
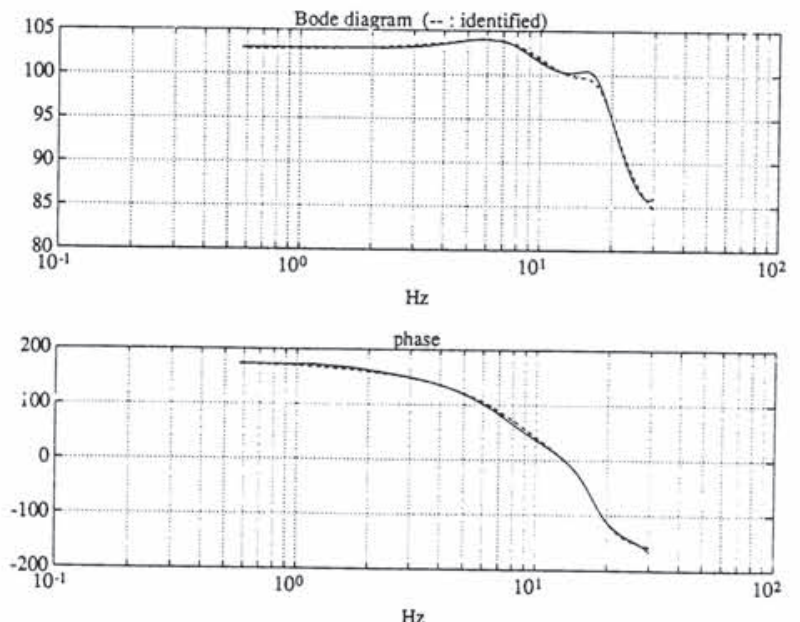

Fig. 6a. "Direct" transfer function $G_{o}(s)$ - Bode diagrams.

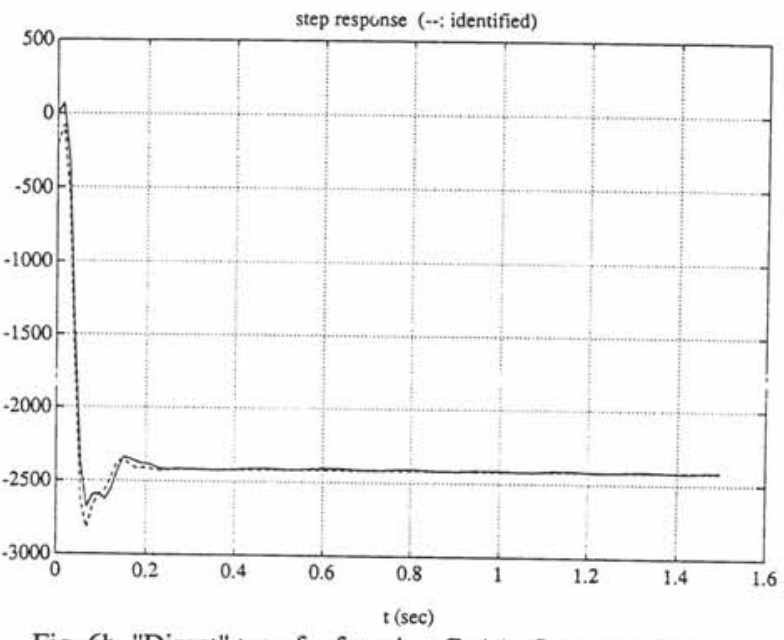

Fig. 6b. "Direct" transfer function $G o(s)$ - Step response.

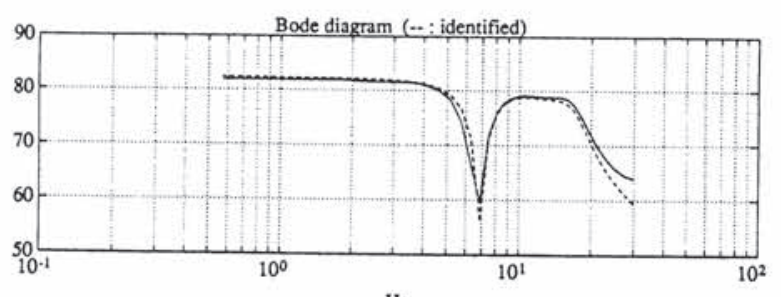

$\mathrm{Hz}$

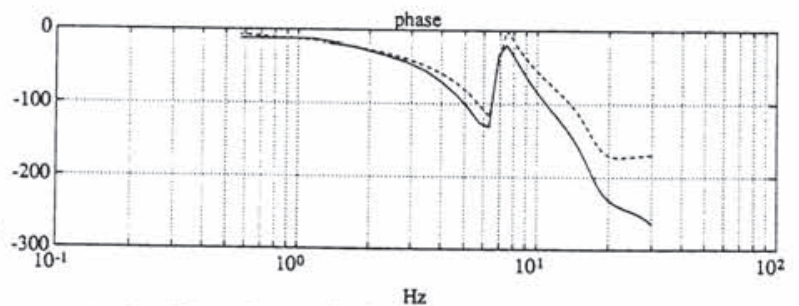

Fig. 6c. "Cross" transfer function $G_{l}(s)$ - Bode diagrams.

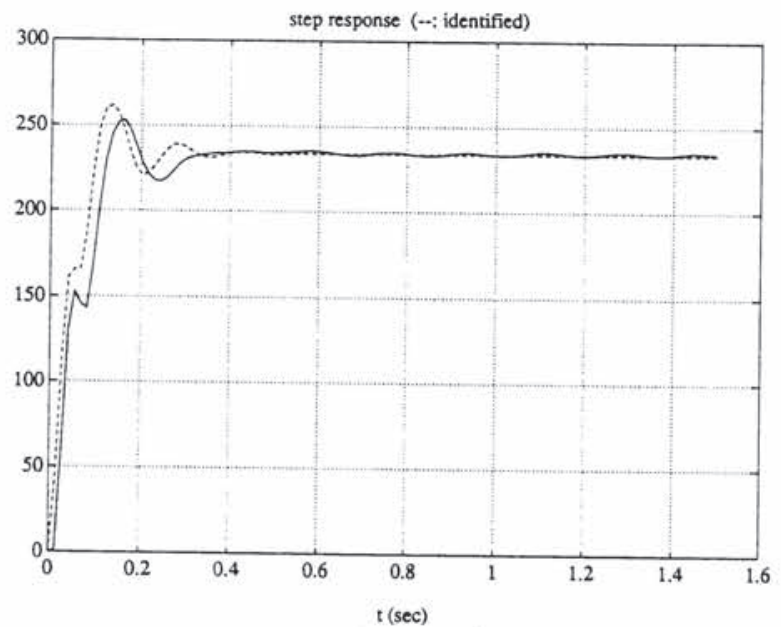

Fig. 6d. "Cross" transfer function $G_{l}(s)$ - Step response. 